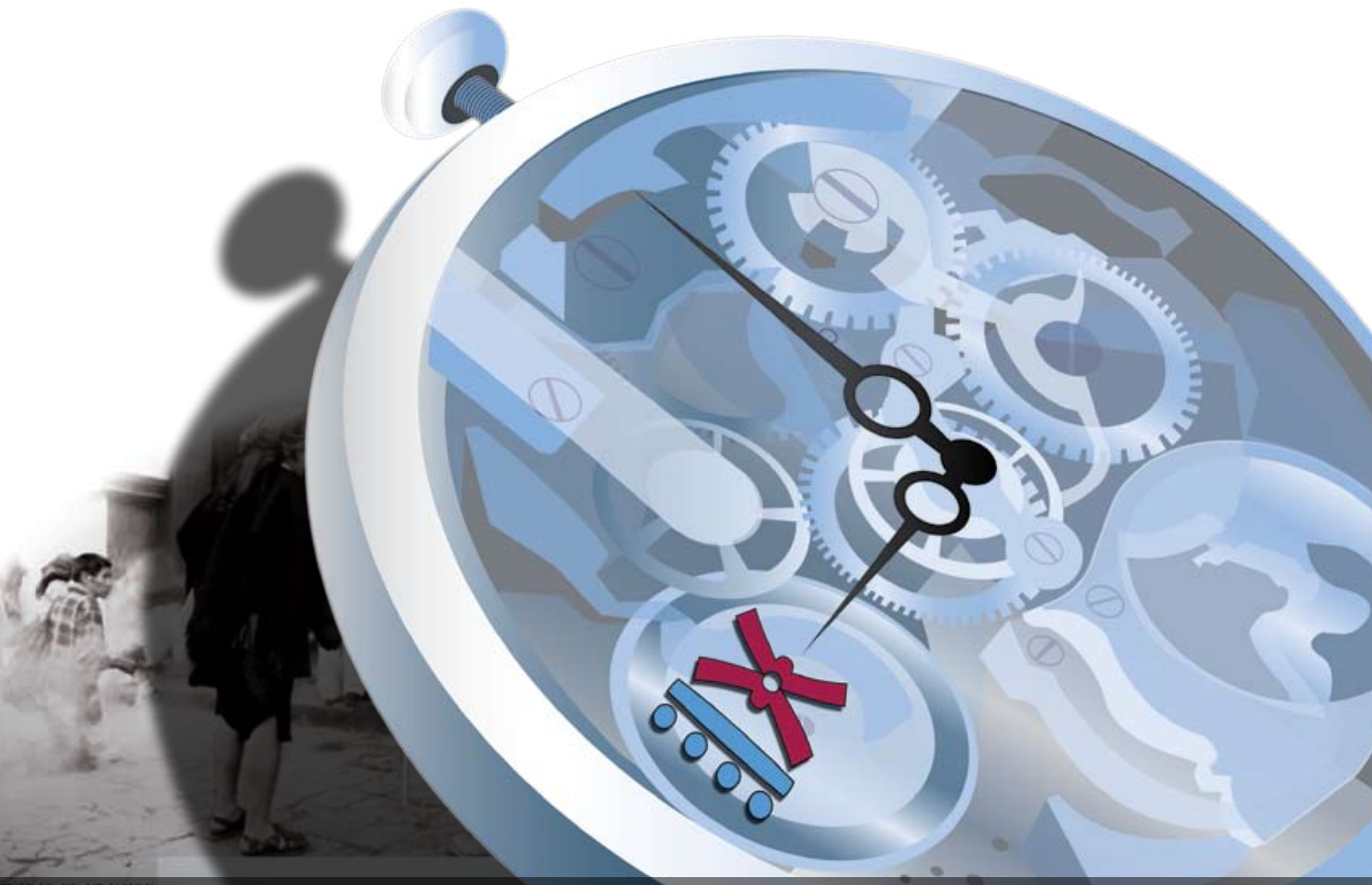

\title{
$9^{\circ}$ C O N G R E S O 2. CENTROAMERICANO DE H ISTO R I A
}

Universidad de Costa Rica

ISSN 1409-469X

Fecha de recepción: 15 de mayo 2008 Fecha de aceptación: 30 de mayo 2008

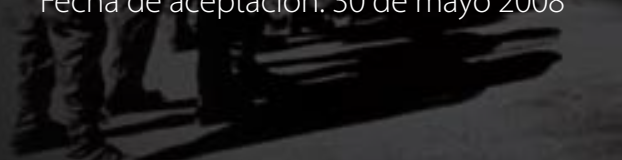

Editores Técnicos:

Allan Fonseca, Andrés Cruz, Gabriela Soto

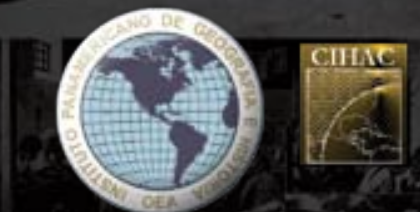

UNA MIRADA COMPARATIVA: LAS GEOGRAFÍAS DE LA MISERIA Y LOS ESPACIOS VERDES EN LAS POLÍTICAS DE LA SALUD EN LA CIUDAD DE BUENOS AIRES A FINES DEL SIGLO XIX

Miembros del Consejo Editorial:

Dr. Ronny Viales, Dr. Juan José Marín 


\section{UNA MIRADA COMPARATIVA: \\ LAS GEOGRAFÍAS DE LA MISERIA Y LOS ESPACIOS VERDES EN LAS POLÍTICAS DE LA SALUD EN LA CIUDAD DE BUENOS AIRES A FINES DEL SIGLO XIX}

Dra. Brisa Varela

Universidad Nacional de Luján Cruce Ruta 5 y 7 Universidad de Buenos Aires- Facultad de Arquitectura,

Diseño y Urbanismo Intendente Güiraldes 2160. Pabellón III Ciudad Universitaria C1428EGA - Buenos Aires.

Lic. Gabriela Campari

Universidad de Buenos Aires- Facultad de Arquitectura,

Diseño y Urbanismo Intendente Güiraldes 2160. Pabellón III Ciudad Universitaria C1428EGA - Buenos Aires. República Argentina. Tel. (54)-11 4209-9465 brivarela@yahoo.com.ar // gecampari@yahoo.com.ar 
Palabras claves: historia ambiental- bordes urbanos- enfermedad- higienismo- espacios verdes.

\begin{abstract}
A partir de las primeras décadas del siglo XIX, la zona sur de la ciudad de Buenos Aires se vio históricamente ligada al Riachuelo y a las actividades de los saladeros y curtiembres, que se encontraban ubicados estratégicamente en las proximidades del Río de la Plata, para facilitar la provisión de agua, evacuación de residuos y transporte por vía fluvial de los productos.
\end{abstract}

Sobre las tierras bajas y mal drenadas aledañas al Riachuelo, se ubicaron los barrios pobres del sur de Buenos Aires, mientras que la barranca, fue el sitio residencial elegido por los más pudientes. Esta relación de pobreza y grandeza, modeló el espacio urbano y fue estableciendo un ordenamiento geográfico en la ciudad que ha mantenido su vigencia a lo largo del tiempo.

En la decisión de aislar, la ciudad quedaba dividida en un "adentro" y un "afuera”, en la cual el área urbana (fundacional, residencial, de paseo, comercial) quedaría limitada por la zona de los alrededores, alejada de la urbe en la cual tendrían lugar las industrias y los servicios como los cementerios, mataderos, ferrocarriles, hospitales y el basural. Por consiguiente estas actividades de servicio que la ciudad expulsaba hacia sus bordes por «sucias» $\mathrm{y}$ «peligrosas», expresaron su presencia en un paisaje urbano imbricado con el desamparo social.

Es en este sentido, que el trabajo orienta su estudio a explicitar los lugares ocupados por los barrios más necesitados del sur de Buenos Aires a fines del siglo XIX y el modelo de ciudad higiénica y promotora de la salud pública, donde además de los pobres la elite instalaba a los anormales al decir de Foucault ([1974-75], 2000): locos, presos, enfermos infecciosos que fueron ocupando el espacio junto a las áreas de servicio como los basurales.

Bajo este contexto quedaron emplazados algunos de los hospitales del servicio médico de la Capital para la asistencia, principalmente, de las enfermedades infecciosas. Los establecimientos fueron concebidos como unidades integradas por un sistema de pabellones distanciados y a la 
vez vinculados entre sí por áreas verdes como medidas higienistas de curación y prevención sanitaria.

Desde el punto de vista metodológico se trabajó con el concepto de triangulación lo que permitió complementar la información de carácter cuantitativa procedente de las estadísticas oficiales con la de tipo cualitativa, proveniente de periódicos, literatura científica de médicos higienistas, y en especial de la lectura de la cartografía histórica, que fue complementada con un minucioso trabajo de campo, en el que hemos localizado diferentes usos y funciones dados a los espacios analizados.

Vemos así que la lectura histórica realizada en el presente de los barrios populares e instituciones hospitalarias del sur de la Ciudad de Buenos Aires, nos permite entender la relación integración/ segregación del área con otros ámbitos internos a la misma. Esta correspondencia de tensión/ colaboración se evidencia en la textura urbana actual y en su relación con un espacio mayor que es el AMBA (Área Metropolitana de Buenos Aires) de la que participa.

\section{El límite sur de la Ciudad de Buenos Aires}

El trazado fundacional de la ciudad de Buenos Aires, que privilegió la topografía del lugar ofreciendo una superficie casi plana, con alturas variables entre los niveles del río, su cercanía a un afluente navegable y la emergente necesidad de disponer de un puerto en el Atlántico, ha ligado históricamente a la ciudad-puerto con el Río de la Plata ${ }^{1}$ y el ordenamiento distintivo entre el "norte” y el "sur”, que fue modelando el espacio urbano hasta la actualidad.

Las primeras iconografías realizadas sobre las características histórico ambientales fueron reflejadas por las representaciones de los viajeros extranjeros, que plasmaron sus impresiones en distintas épocas de la región, como fueron las de Ulrico Schmidel, a través de sus crónicas registradas en la participación de la armada de Pedro de Mendoza en 1536 durante la primera fundación de Buenos Aires. (Fig. 1)

$1 \quad$ El nombre con que lo distinguían los pueblos originarios fue Paraná Guazú que significaba "río semejante al mar". 
Hacia el primer centenario de las revoluciones por la independencia , en 1910, Denis expresaba: "El terreno donde se ha edificado la ciudad es una meseta regular de $20 \mathrm{~m}$ de altitud, fragmentada por valles de fondo playo inundables (bañados). El Riachuelo en la desembocadura de uno de esos valles, ha ofrecido a Buenos Aires su primitivo puerto. Las tierras bajas, mal drenadas de los valles han sido ocupadas por los barrios más pobres. Sus laderas "las barrancas", son, por el contrario, un sitio de residencias aristocráticas.” (Denis, [1920] (1987): 315) (Fig. 2)

Es en este contexto de miseria y grandeza que la estructura socio-espacial y socioeconómicapolítica de la ciudad fue estructurando el espacio geográfico del sur.

A partir de las primeras décadas del siglo XIX, la zona sur se vio ligada al Riachuelo, en el cual tuvieron lugar la instalación de los saladeros, curtiembres y fábricas de sebo, ubicados estratégicamente por la facilidad de provisión de agua y evacuación residual de sus desechos, conformando a su vez, el circuito por el que se trasladaba por vía fluvial la producción al puerto para ser luego exportada. Estas industrias se desarrollaron marcadamente a lo largo de los cincuenta años entre 1810 y 1860, siendo su producción (tasajo) enviada especialmente a Brasil y Cuba en calidad de alimento para la población esclava.

A mediados de la década del '70, producto de las normativas sanitarias implementadas en la ciudad y las modificaciones del mercado internacional por el consumo de las carnes enfriadas, se produjo la depresión en la economía del tasajo, lo cual llevó a que estos terrenos del sur, pasaran a ser quintas para vacacionar como la de "Anderson” en el meandro sur del Riachuelo, «El Rincón» y la de "Ochoa” construida en lo que fuera el saladero el «Mirador».

En paralelo al decaimiento de estas actividades, la cría del ganado para el consumo de la ciudad se realizaba fuera de sus límites pero la matanza seguía dentro de ellos (en Barracas y Parque de los Patricios). La zona sur debió ser interconectada para el pasaje a pie del ganado y para el traslado de hortalizas y producción láctea desde la campaña sur hacia la ciudad. Las conectividades entre la ciudad de Buenos Aires y lo que sería el conurbano bonaerense, quedaron impulsadas por las construcciones que se realizaron sucesivamente sobre el Riachuelo a partir de 1791 de los puentes Gálvez, Paso de Burgos (hoy Uriburu), Pueyrredón y de la Noria que mejoraron la 
comunicación entre ambas orillas.

A partir de 1872 se trasladó definitivamente el Matadero del Sur desde la zona de BarracasConstitución a Parque de los Patricios, época en la que además múltiples riachos recorrían la zona desembocando en el Riachuelo. Mientras tanto se iba poblando la orilla sur de este curso generado por las actividades de curtiembre y frigoríficas, las cuales hicieron que dos años más tarde, el gobierno provincial aprobara la traza de un nuevo pueblo: Valentín Alsina (de 100 ha) y solicitara la concesión de una línea de tranvías para conectar el pueblo con los corrales "del otro lado" (en Parque de los Patricios).

Según los datos estadísticos, la población de la ciudad de Buenos Aires que se estimó en 1799 2 en 40.000 habitantes y en 1824 en 81.000; creció geométricamente en la segunda parte del siglo XIX como demuestran los censos: 177.000 en 1869; 433.000 en 1887; 663.0000 en 1895 y 1.575.000 en 1914, en gran medida por el flujo migratorio de origen europeo, que se ubicó principalmente, al sur de Buenos Aires en los barrios de la Boca, Barracas, Constitución y San Telmo constituyendo estos inmigrantes recién llegados, los sectores populares de la ciudad.

Fue en estos barrios del sur de Buenos Aires, en los cuales tuvieron lugar las geografías de la miseria, el aislamiento sanitario para las epidemias e insanos, y la disposición de la basura al sudoeste de la ciudad, en el barrio Parque Patricios, donde ya funcionaba el Matadero del Sur.

\section{Las geografías de la miseria: el hospital y la higiene urbana}

A partir de la segunda mitad del siglo XIX , tras la conmoción generada por las epidemias acontecidas de cólera (1869) y fiebre amarilla (1871) poco comunes en Buenos Aires, y después de la sanción de la Ley de Federalización de Buenos Aires (1880), la ciudad adoptó como proyectos de higiene urbana, un programa de salud preventiva y social de la población que habrían de regir las condiciones de salubridad en el ambiente urbano.

Estas medidas se vieron enfatizadas a partir de la primera Intendencia Municipal de Buenos Aires de Torcuato de Alvear (1883-1887), en la cual se proyectaron los programas que tuvieron 2 por Azara 
a cargo el servicio y la calidad del agua potable, el tratamiento e incineración de los residuos, el saneamiento cloacal, las inspecciones sanitarias de los mataderos, cementerios; como así también, la habilitación de hospitales y construcción de establecimientos sanitarios, especialmente para el tratamiento de las enfermedades infecciosas.

Estos programas entroncan en la concepción higienista adoptada de los modelos europeos de los reformistas sociales y políticos (Owen [1816], Fourier [1829] y Garnier [1917] 1991), cuyo pensamiento se orientaba a elevar la calidad de vida de los habitantes, y a obtener mejores respuestas a los problemas de hacinamiento y enfermedades epidémicas que habían repercutido a nivel mundial.

En este sentido, la salud pública dependería según afirmaba Eduardo Wilde, de la suma de la salud individual que se lograría potenciando la acción de factores naturales “(...) la acción benéfica de todos estos agentes: el aire, el agua, los alimentos, el vestido, la habitación, la desecación del suelo, los desagües (...) provision de agua, su medio de desinfección de los pozos donde van los líquidos servidos, su extracción de basuras (...)” (Wilde: 1887, 55-56). Diseñar una ciudad en la que estos agentes intervendrían se constituye en un eje a considerar en la concepción y formulación de las instituciones hospitalarias y educativas.

En los estudios de Foucault ([1966]2003; [1980]1996), el autor planteó la concepción de hospital como medida higiénica, en el cual la institución se tornaba indispensable como protección de los sanos contra la enfermedad, de los enfermos contra las prácticas de la gente ignorante y entre los mismos enfermos. También analizó su relación con los espacios concretos que le fueron asignados a la enfermedad en la ciudad y la institucionalización de una medicina colectiva, social y urbana en el discurso político y el saber científico.

A su vez, Buenos Aires evidencia una disposición a la intervención del Estado sobre el espacio público. Implementada a través de la erradicación de focos de contaminación mediante acciones de: "aislamiento" como medida profiláctica de "la enfermedad" en la ciudad, y de saneamiento y desinfectación, a partir del alejamiento y/o destrucción de los sitios u objetos contaminados, y/o cremación cadavérica obligatoria para los cadáveres no reclamados y para aquellos parientes que así lo deseaban. 
Para el caso de los dementes, el aislamiento estuvo dado por la reclusión en establecimientos llamados manicomios, basados en la organización del hospicio mediante la distribución y separación de los "insanos" por departamentos en relación con el grado de agresividad en su conducta evaluada desde el poder médico, o bien si su procedencia estaba ligada a causas penales.

Estas medidas higienistas concibieron los hospitales como unidades integradas por un sistema de pabellones distanciados y a la vez vinculados entre sí por áreas verdes. Esta organización debía permitir la articulación de la dinámica grupal o individual de cada pabellón, por eso cada construcción debía contar con los servicios necesarios para el desenvolvimiento propio. El asilamiento entre pabellones y con el exterior se debía complementar a través de arboledas perimetrales, a diferentes alturas a fin de conformar barreras o filtros vegetales para purificar la atmósfera, higenizar el ambiente y embellecer el entorno hospitalario. Si bien desde el punto de vista edilicio, varios de los hospitales adecuaron sus instalaciones a estos principios, otros, sin embargo tuvieron que adaptarse a las realidades económicas que estaban a su alcance.

En la decisión de aislar, la ciudad quedaba dividida en un "adentro" y un "afuera”, en la cual el área urbana (fundacional, residencial, de paseo, comercial) quedaría limitada por la zona de los alrededores, alejada de la urbe en la cual tendrían lugar las industrias y los servicios como los cementerios, mataderos, ferrocarriles, hospitales y el basural. (Fig. 3)

Por consiguiente estas actividades de servicio que la ciudad expulsaba hacia sus bordes por "sucias” y “peligrosas”, expresaron su presencia en un paisaje urbano imbricado con el desamparo social que, con extrema crudeza, narró Martínez Estrada en los años treinta: "Pobreza y fealdad (que) se apelotona a las puertas de Buenos Aires, como pordioseros a la puerta del palacio. Maderas y latas con charcas verdosas y basuras. Son los desechos de la metrópoli y al mismo tiempo un montón de escombros de suelos de opulencia; lo que no quiere ser ciudad y queda recalcitrante fuera del municipio; y al mismo tiempo lo que ya no quiere ser soledad y se apeñusca en los límites de la campaña. Por eso tales viviendas sórdidas y feas simultáneamente son las dos cosas: la ciudad y el campo. Mirándolas bien se ve que son ranchos que se han deslizado desde el fondo de las llanuras, amontonados a la orilla del mundo moderno. La casa de cinc que en una o dos habitaciones reúne la familia numerosa es un rancho que ha cambiado los materiales de 
construcción; el barro por el metal, como antes el cuero por el barro. Al adobe lo ha reemplazado la chapa, con lo que se ha hecho menos estable. Materiales heterogéneos, desperdicios arrastrados en la tarea de la hormiga y puestos ahí para llenar intersticios y aparentar solidez. La verdad es otra: un rancho provisorio, portátil que puede desarmarse lámina a lámina, como se construyó y transportarse (...) Casas que son, dirá, "refugios pero no hogares", signadas por lo efímero, lo no consolidado, lo inestable, en las que el ladrillo se "desmenuza antes que la carne" de quien lo habita. Ese lugar de la pobreza es también para el autor, el lugar de "la crueldad, la falsedad y el rencor, hacia otros o para consigo” (Martínez Estrada, [1953]1957): 261-262).

En el marco de esta dinámica urbana, se regularizaron y ampliaron las intervenciones públicas en los establecimientos para la salud que funcionaban precariamente, a partir de la institucionalización de la Asistencia Pública (1883) y la Administración Sanitaria como institución conexa (1892), a fin de centralizar la dirección científica de los hospitales y los servicios de beneficencia como prioridades sanitarias en sus aspectos físicos, mentales y sociales de los habitantes del municipio, y en especial, de aquellos en situación de pobreza.

Luego de estas instancias, nuevos fenómenos han conformado la "Medicina de Estado" (Foucault, [1980]1996) en la cual el poder político fue acumulando una serie de procedimientos y conocimientos para hacer más efectivo su ejercicio mediante: la normalización de la enseñanza médica universitaria, su ejercicio social y el nombramiento de médicos para ocupar cargos en la función pública como lo fue el caso de los médicos argentinos Guillermo Rawson, Eduardo Wilde, Emilio Coni, José Penna entre otros profesionales y técnicos que actuaron promulgando la "cultura de la higiene".

Desde la perspectiva de Armus (2007) esta cultura de la higiene articuló normas cotidianas en la ciudad que fomentaron a través de la instrucción un cambio social de conductas, que facilitaba el reconocimiento y la integración en la sociedad.

Bajo este contexto quedaron emplazados al sur de la ciudad, algunos de los establecimientos del 
servicio médico de la Capital como los Hospitales Rawson (Fig. 4), Muñiz (Fig. 5) y Argerich pertenecientes a la Asistencia Pública, el Hospital Militar Central y el Hospicio de las Mercedes de jurisdicción nacional, el Hospital Inglés como establecimiento extranjero y el Hospital de Alienadas y la Casa de Expósitos a cargo de la Sociedad de Beneficencia, función que había sido delegada por el Estado a estas organizaciones benéficas para la asistencia especialmente, de mujeres y niños.

El Hospital Nacional de Alienadas (1898) llevó adelante los primeros servicios sobre los terrenos de la antigua Convalecencia a partir de 1854, a las mujeres dementes que procedían del Hospital de Mujeres, las detenidas en la Cárcel o encontradas en la vía pública en estado de abandono. Por otra parte la Asilo o Casa de Expósitos fue fundada 1779, trasladándose definitivamente al actual barrio de Barracas en 1873 bajo el nombre de "Casa Cuna” para la asistencia y protección de la niñez desvalida.

El Hospicio de las Mercedes (ex Hospicio de la Buena Ventura) se fundó con el nombre de Casa de Dementes en el año 1863, con el propósito de instalar fuera de la ciudad una institución que albergara a los hombres "insanos", privilegiando una ubicación alejada del centro y de fácil llegada y cercanía a las vías del ferrocarril. El establecimiento estaba dividido por departamentos (pensionistas, locos tranquilos, epilépticos y paralíticos, furiosos, semi-agitadores y destructores, insanos criminales y convalecientes) para el cual, la Comisión de Inspección Hospitalaria inició las observaciones necesarias para que se contara con jardines exteriores entre el muro de circunvalación y los edificios, a fin de adecuar la institución según los criterios higienistas.

El Hospicio de Inválidos (llamado posteriormente Hospital Mixto 1884) fue fundado en el año 1868 para asistir a los excombatientes de la guerra contra Paraguay. Debido a la necesidad de contar con instituciones sanitarias en 1889 el hospital cambió de nombre por el de Rawson, reubicando su función de asilo en otro terreno arrendado por el Municipio. Este establecimiento fue el primero en el tratamiento de vías urinarias, servicio al cual complementó la asistencia de los consultorios externos, maternidad y cirugía.

En 1882 se creó la Casa Municipal de Aislamiento (denominado posteriormente Hospital "Doctor Francisco Muñiz") como establecimiento especializado para asistir las enfermedades 
contagiosas, dado que el "Hospital de Hombres (hoy de Clínicas) no podía cubrir con las necesidades demandadas y había sido decretada su clausura. Por otra parte el "Lazareto San Roque” debía prestar sus servicios como hospital común en vez de recibir dolencias epidémicas, por consiguiente, la institución comenzó sus servicios sanitarios para tratar la varicela considerada la enfermedad "vergonzosa de América" en ese entonces. Según las estadísticas (Municipalidad de la Capital, 1910) de las enfermedades infecciosas más frecuentes registradas en el hospital durante el período (1883-1910) se encontraron: sarampión (especialmente en las estaciones de invierno y primavera), tuberculosis pulmonar, viruela, fiebre tifoidea y difteria entre otras.

Cabe mencionar en esta instancia, que teniéndose en cuenta el modelo de aislamiento que había sido adoptado en la ciudad, a su parecer, el Doctor José Penna no dejó de lado la relación existente entre el estado irreversible de los enfermos que padecían estas enfermedades y la falta de recursos económicos que los ligaba a la asistencia pública, por lo que argumentó al respecto: “(...) cuando la enfermedad es de marcha prolongada como sucede con las infecciones crónicas, entonces el aislamiento pierde toda su importancia (...) Lo tenemos bien comprobado en los pobres tuberculosos y leprosos de la Casa de Aislamiento. La desgracia, la pobreza, el abandono de los amigos y parientes los deja en la soledad más detestable (...) el aislamiento de la enfermedad, es aceptado sólo en el hospital especial como un último extremo, como una necesidad fatal (...) no les queda materialmente otro recurso que acogerse á este tranquilo y humanitario santuario que la provisión municipal le ha erigido.” (Municipalidad de la Capital, 1910: 110).

Por otra parte, el Hospital Militar Central fue inaugurado en 1889, con la finalidad de destinar la asistencia a "sus enfermos", los cuales venían siendo asistidos en los hospitales para "civiles”. La institución se conformó a partir de un sistema de construcción de pabellones aislados unidos por amplias galerías cubiertas y separados por espaciosos jardines. El establecimiento militar contó con un departamento de baños (inmersión, turcos, piscina) y con el servicio de dos ambulancias automóviles únicas en la ciudad.

A su vez el Hospital “Doctor Cosme Argerich” empezó a funcionar como Estación Sanitaria (1897), especialmente prestando asistencia orientada a la clínica médica y cirugía, derivando todos los cuadros epidémicos a la Casa de Aislamiento para su intervención. 
Como consecuencia de la creciente demanda de asistencia hospitalaria a nivel nacional y las limitaciones de espacio higiénico para la admisión de enfermos provenientes de distintas provincias de la república, que los establecimientos de la ciudad de Buenos Aires presentaban como fue el caso del Hospicio de las Mercedes, se formó la Comisión de Asilos y Hospitales Regionales, con el objeto de realizar un plan sanitario, para el cual se designó al médico correntino Doctor Domingo Cabred para presidirla y aplicar las reformas correspondientes.

El plan no contemplaba las obras necesarias para ampliar el servicio sanitario de la ciudad de Buenos Aires, sino evaluar la necesidad por provincia, destacando las condiciones ambientales de cada región del país a fin de corresponder a un tratamiento adecuado según la enfermedad en relación al clima, luz o altura. En el mismo se resaltó la necesidad de que "el sitio en el cual debían levantarse los hospitales o asilos fue una de las primeras preocupaciones, pues no sólo debían tenerse en cuenta las necesidades de la zona a que iban a servir, sino que reunieran las condiciones apropiadas, según su naturaleza, la llanura, la montaña, facilidades de acceso, condiciones de clima, fertilidad del suelo, e hidrología, superficial o subterránea”. (Previsión y Asistencia Social, 1917: 14-15).

Este plan reformista sanitario enfatizó la creación de los grandes hospitales comunes, bajo el concepto de una “aislada población de sanidad”, que debía emplazarse entre jardines, árboles frondosos y amplios prados, siguiendo la misma concepción rectora de higiene para la conformación de los conjuntos hospitalarios.

Las instituciones sanitarias emplazadas al sur de la ciudad, siguieron funcionando ininterrumpidamente, recibiendo enfermos de diversos puntos del país y ampliando día a día la demanda asistencial. Sus propias dinámicas y la relación con el entorno hizo que se conformara un paisaje diferenciador, el cual fue respondiendo a los movimientos funcionales del sector que ligaban esta zona de servicios, con la modernizada ciudad de Buenos Aires.

Este paisaje, producto de la yuxtaposición de un conjunto de formas heterogéneas, de tiempos históricos representativos de diversas maneras de producir y construir el espacio (Santos, 1996), 
transparentó una lectura sujeta a la enfermedad, miseria y aislamiento de los que padecen detrás del muro de la mirada.

Ese sur “peligroso” también contenía el Cuartel de Infantería junto a los Talleres de Arsenal de Guerra, la Casa Correccional de Menores, el ex Cementerio del Sud, los parques (Lezama, Ameghino, Patricios, Pereyra), plazas (Constitución, Herrera, España, Virrey Vértiz), jardín botánico, casas obreras, estaciones de ferrocarril coexistiendo funcionalmente en la trama octogonal de la ciudad, entre quintas y testamentarias que fueron reflejo del domino privado de la tierra en otros tiempos.

En relación con la disponibilidad y valor de las tierras, pero también en función de una ideología elitista, este fue el espacio geográfico que Buenos Aires le asignó a la basura que se producía en ella.

Desplazada desde el centro al sudoeste de la ciudad, por el escaso valor comercial de estas tierras, la baja densidad de población, y el emplazamiento apartado con respecto al casco fundacional que se hacía necesario para hacer desaparecer los quemaderos por las proximidades de las edificaciones. Si bien estas condiciones primaron la elección del lugar, contrariamente a éstas, la zona céntrica había aumentado considerablemente su población y la valoración edilicia de los "huecos" ${ }^{3}$ había quedado sujeta a las reformas urbanas que justificaban el traslado de los basurales.

A pocas cuadras del Hospital Muñiz, Rawson, Hospicio de las Mercedes y del Parque de los Patricios quedó instalado el predio para la deposición de la basura a mediados de 1860. El tratamiento de los residuos estaba compuesto por la separación de materiales recuperables, la quema posterior mediante la utilización de hornallas a cielo abierto y un servicio del tren carguero que llevaba la basura y acarreaba el carbón. Los restos de huesos y carne se los apartaba y eran llevados a los galpones de la “fábrica” para extraerles la grasa por cocción.

3 Denominado al terreno de poquísimo precio que siendo o no de dominio particular, con deudas o libres de ellas originarias de la repartición de tierras en la época fundacional, no se cultiva ni tiene provecho alguno. 
Este sistema de tratamiento de residuos urbanos, trajo aparejado la relación incondicional de marginalidad de los sectores más deprimidos de la sociedad, que a través del "cirujeo" separaban de la basura aquello que podían darle uso personal o de reventa.

\section{Los espacios verdes en la política de la salud}

Los conceptos del higienismo se incorporaron a la planificación paisajística de Buenos Aires, a través del “árbol” como instrumento sanador de la urbe. Esta concepción primó en la intervención de espacios públicos, como el arbolado de aceras en calles y avenidas, jardines de los hospitales, plazas y parques, bajo una noción organicista de la trama urbana, en la cual se consideraba a la ciudad como un "organismo vivo" que respiraba a través de ellos.

Como parte de la política de la salud, desde la Intendencia Municipal se incorporaban estos nuevos conceptos que resaltaban la importancia de los espacios verdes en una ciudad moderna, con alta densidad poblacional y actividades industriales emergentes, a los cuales no se les podía “ (...) aplicar el calificativo de lujo, sino de higiene pública, y todas las ciudades (...) Europa, las de América misma, poseen grandes centros de esta clase que sirven de desahogo a la población, tanto más hoy que por el precio de la tierra se va estrechando la habitación en el hogar.” (Alvear En: Beccar Varela, 1926: 207)

Desde fines del siglo XIX este discurso biológico, como señalara Armus (2007), promulgaba un programa regeneracionista que apuntaba a equiparar la ciudad con espacios verdes para advertir su beneficio en el ambiente urbano, cada más acelerado e industrial, en la prevención de las enfermedades contagiosas como la tuberculosis.

Este aspecto utilitario e higiénico, relacionaba la necesidad de implementar estas áreas como grandes "pulmones de la ciudad"; dado que "si el medio crea a la especie, como tan justamente lo sostienen los naturalistas, el medio social formará al individuo, y siendo las grandes ciudades otros tantos centros de aglomeraciones humanas y el medio donde se desenvuelve la civilización, justo es que se procure mejorar ese ambiente a fin de que los habitantes encuentren las mayores comodidades y el bienestar necesario para la vida” (Carrasco, 1924: 135)

Para lo cual la ciudad debía ser pensada como un todo, donde se integrarían los aspectos sociales y de diferenciación y jerarquización en las escalas de los espacios abiertos, los cuales se hacían necesarios por estética, higiene, expansión y solaz. 
Los espacios verdes, dentro de la perspectiva higienista, habrían de proporcionar un ambiente adecuado, luminoso, el cual permita ver el sol estimulando el esparcimiento de la sociedad, en donde se pueda encontrar sombra y frescura en el verano y sol y reparo en el invierno, constituyendo simultáneamente en elementos de decoración e higiene urbana.

En este sentido los espacios verdes públicos se intervinieron a partir de una idea rectora que consistía en la incorporación de especies vegetales, especialmente arbóreas, y coberturas de césped, como elementos compositivos primordiales que reforzaban el pensamiento ligado a la valorización de los espacios verdes, en relación con el aporte de oxígeno diario que la vegetación aporta a la atmósfera, función que resultaba esencialmente adecuada, para promover la calidad de vida de las personas al servicio del bienestar humano desde un modelo de ciudad higiénica. Para el ambiente hospitalario, la incorporación del verde en los jardines de las instituciones asistenciales, si bien, por un lado reforzaba y materializaba la idea del aislamiento; por otro, el entorno vegetal proporcionaba a los enfermos un entorno natural oxigenante y estético que estimulaba las visuales de los jardines a través de las habitaciones, y proporcionaba a su vez, sitios agradables donde pudiesen reposar durante el día, aguardando la curación y el alta hospitalaria.

\section{Consideraciones finales}

La lectura de las disposiciones espaciales de la histórica Buenos Aires, que aún perviven en el sur poco modernizado y degradado de la ciudad, nos permiten conceptualizar esa particular construcción sociopolítica del espacio urbano como una geografía de la vulnerabilidad y leer la producción social de los espacios verdes asociados con la salud, dentro de la relación de pobreza/ elite, que fue modelando el espacio urbano, estableciendo un ordenamiento geográfico que ha perdurado en la ciudad a lo largo del tiempo.

También articular la disposición espacial de los barrios de la pobreza, con las actividades económicas relacionadas con el Riachuelo y la desvalorización de los terrenos. Es en ese contexto en el que quedaron aislados aquellos hospitales de la asistencia de la Capital que debían responder a un programa sanitario a fin de garantizar la evacuación de los problemas de insalubridad y hacinamiento que amenazaban las zonas urbanas más pobladas, pero también desde un criterio de segregación urbana. 
Por último diremos que en el actual proceso político que se vive en la ciudad de Buenos Aires se genera una polémica en torno a la revalorización de los terrenos del sur para megaproyectos, desde nuestro punto de vista es central, desde una mirada socialmente integradora, propiciar el rescate patrimonial de aquellas intervenciones públicas que hacen a una historia pero también a un presente y un futuro que aún se columpia entre la inclusión o la exclusión de los barrios del sur. 


\section{Bibliografía}

Armus, Diego (2007) La ciudad impura. Salud, tuberculosis y cultura en Buenos Aires, 1870-1950. Buenos Aires: Edhasa.

Beccar Varela, Adrián (1926) Torcuato de Alvear-Primer Intendente Municipal de la Ciudad de Buenos Aires - Su acción edilicia. Buenos Aires: G. Kraft Ltda. S.A. de Impresiones Generales.

Boffi, Luis (s/f) Hospitales de la Ciudad de Buenos Aires. Su pasado, presente y futuro. Hospital Torcuato de Alvear. Cuadernillo de la Institución.

Carrasco, Benito (1924) Parques y Jardines. Buenos Aires: Editorial Peuser.

Denis, Pierre [1920] (1987) La valorización del país. La República Argentina-1920. Buenos Aires: Editorial Solar.

Foucault, Michel ([1966] 2003) El nacimiento de la clínica. Una arqueología de la mirada médica. Trad. Francisca Perujo. Buenos Aires: Siglo XXI Editores.

Foucault, Michel ([1974-1975] 2000) Los anormales. Buenos Aires: Fondo de Cultura Económica.

Foucault, Michel ([1980] 1996) La vida de los hombres infames. Barcelona: Ariel S.A.

Gobierno de la Ciudad de Buenos Aires (GCBA) (2005) Buenos Aires hace cien años, a través de las postales. Buenos Aires a hundred years ago, though its postcards. Secretaría de Cultura: Comisión para la Preservación del Patrimonio Histórico Cultural de la Ciudad de Buenos Aires.

Martínez Estrada, Ezequiel ([1953] 1957) Radiografía de la pampa. Buenos Aires: Losada. 
Municipalidad de la Capital (1910) La Administración Sanitaria y Asistencia Pública de la Ciudad de Buenos Aires. Dir. Penna. J. Tomo II. Buenos Aires: Imprenta Litográfica y Encuadernación de G. Kraft.

Owen [1816], Fourier [1829], Garnier [1917], Le Corbusier [1922] (1991) Ciudad y utopía. Buenos Aires: Centro Editor de América Latina.

Previsión y Asistencia Social (1917) Asilos y Hospitales Regionales en la República Argentina. Publicado en el diario “La Nación”. Buenos Aires 9 de marzo.

Prignano, Angel (1999) Basuras, roñas y otras fetideces. 400 años de residuos en Buenos Aires. En: Todo es Historia $\mathrm{N}^{\circ}$ 387. Buenos Aires.

Santos, Milton (1996) Metamorfosis del espacio habitado. Barcelona: Oikos-Tau.

Taullard, Alfredo (1927) Nuestro antiguo Buenos Aires. Como era y como es desde la época colonial hasta la actualidad. Buenos Aires: Editorial Peuser.

Wilde, Eduardo (1887) Arrendamiento de las obras de salubridad de la capital. Buenos Aires: Imp. Tribuna Nacional.

\section{Cartografía}

Plano de la Ciudad de Buenos Aires (1866) Indicando las líneas de Ferro-Carriles y Trenways en exploración y proyecto, según los documentos especiales suministrados por las Companias, levantado por el Ingeniero $\mathrm{D}^{\mathrm{n}} \mathrm{A}$. Aymez. Escala de la copia 1:1. Buenos Aires: Lit. J. Pelvitain.

Guia Kraft (1888) IV Año, $1^{\text {er }}$ Trimestre. Escala de metros 1:10.000. Buenos Aires: Lit. é Imp. G. Kraft.

Plano de la Ciudad de Buenos Aires Capital de la República Argentina (1904) con el trazado 


\section{9 $9^{\circ}$ CONGRESO \\ g) CENTROAMERICANO \\ DL IIISIOIR}

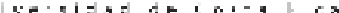

Indexaciones: Repositorio de Revistas UCR, DIALNET, Latindex, REDALYC Directorio y recolector de recursos digitales del Ministerio de Cultura de España, Directory of Open Access Journals.

Diálogos Revista Electrónica de Historia ISSN 1409-469X. Número especial 2008. Dirección web: http://historia.fcs.ucr.ac.cr/dialogos.htm

general de calles confeccionado por el Departamento de Obras Públicas de la Municipalidad. Escala M.0.001 m/m=28 ms. Buenos Aires.

Plano de la Ciudad de Buenos Aires Capital de la República Argentina (1907) con el trazado general de calles confeccionado por el Departamento de Obras Públicas de la Municipalidad. Escala de metros 1:10.000. Buenos Aires: Grabado e impreso en los Talleres de $\mathrm{G}^{\mathrm{mo}} \mathrm{Kraft}$.

\section{Figuras}

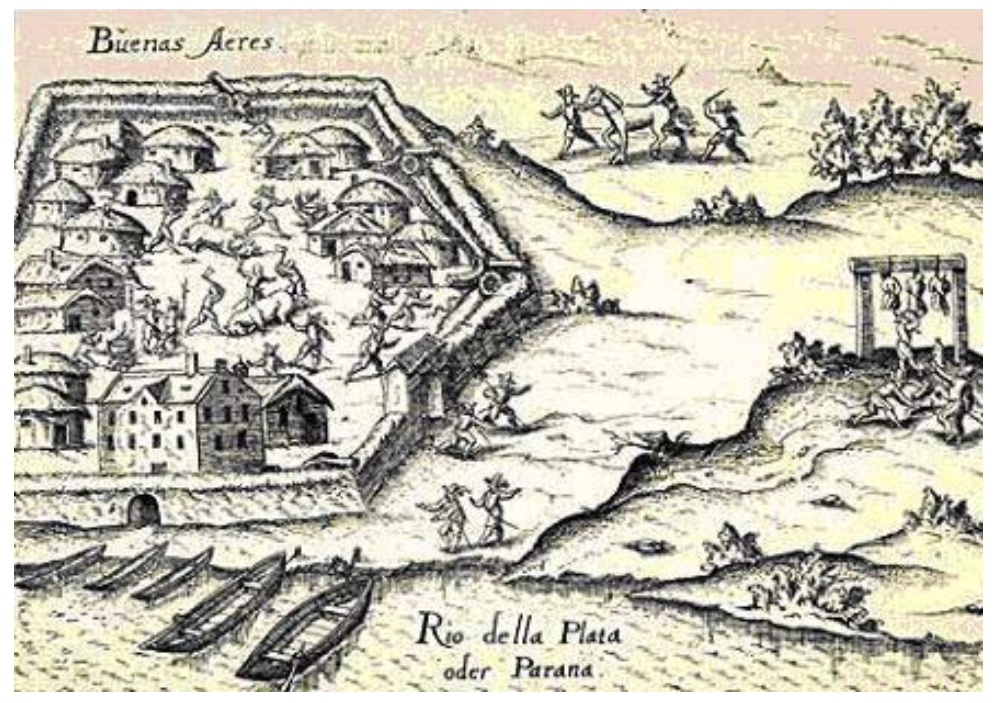

Fig. 1 Junto a la orilla del Río de la Plata, el fuerte de Buenos Aires. (Schmidel siglo XVI) 


\section{9) $99^{\circ}$ CONGRESO \\ of $\mathrm{S}$ CENTROAMERICANO \\ OJ UL IIISIORIA}

Indexaciones: Repositorio de Revistas UCR, DIALNET, Latindex, REDALYC Directorio y recolector de recursos digitales del Ministerio de Cultura de España, Directory of Open Access Journals.

Diálogos Revista Electrónica de Historia ISSN 1409-469X. Número especial 2008. Dirección web: http://historia.fcs.ucr.ac.cr/dialogos.htm

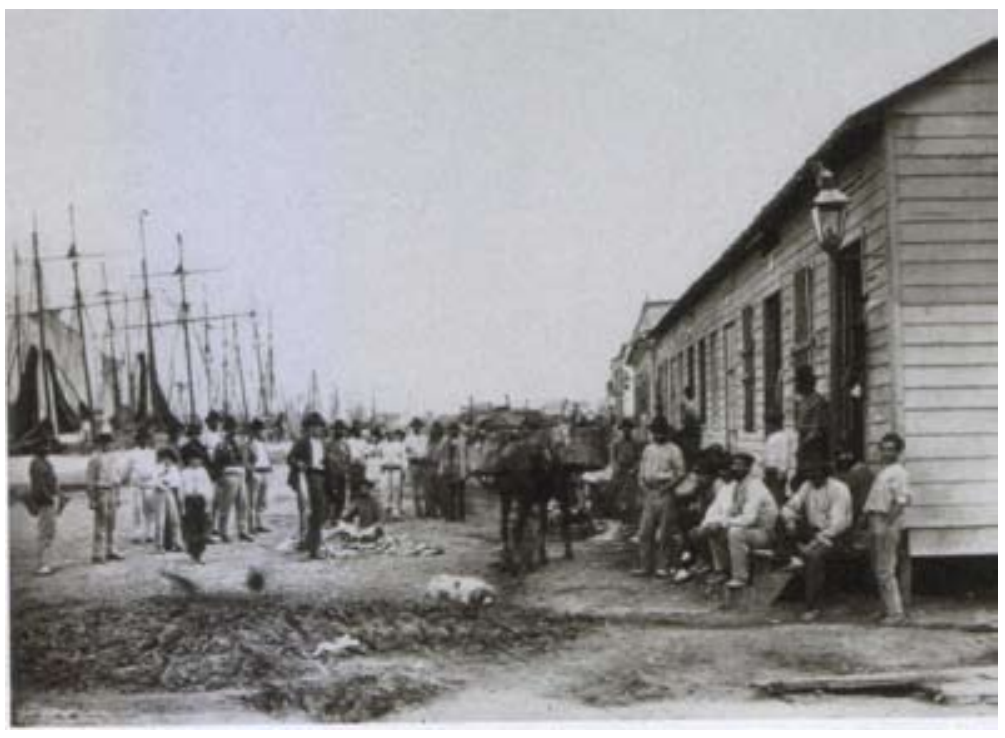

Fig. 2 Viviendas precarias en la boca del Riachuelo 1877 (Archivo General de la Nación)

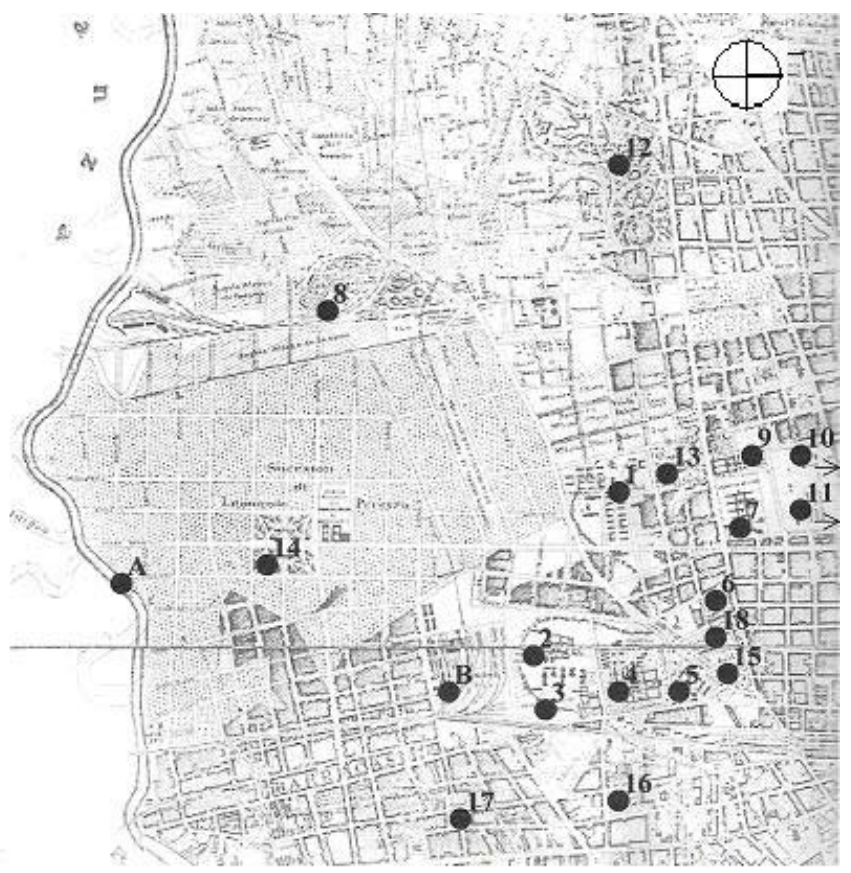

Fig. 3 Plano de la Ciudad de Buenos Aires Capital de la República Argentina 1907 
(Instituto Histórico de la ciudad de Buenos Aires) Referencias: 1-Hospital Muñiz 2- Hospital Nacional de Alienadas 3-Asilo de Expósitos 4-Hospicio de las Mercedes 5- Hospital Rawson 6-Hospital Inglés 7-Hospital Militar Central 8-Quema de basuras 9-Casa Correccional de Menores 10-Cuartel de Infantería 11-Talleres de Arsenal de Guerra 12-Parque de los Patricios 13-Parque Ameghino 14-Parque Pereyra 15-Plaza España 16-Plaza Virrey Vértiz 17-Plaza Herrera 18-Jardín botánico A-Riachuelo B-Talleres del Ferrocarril del Sud

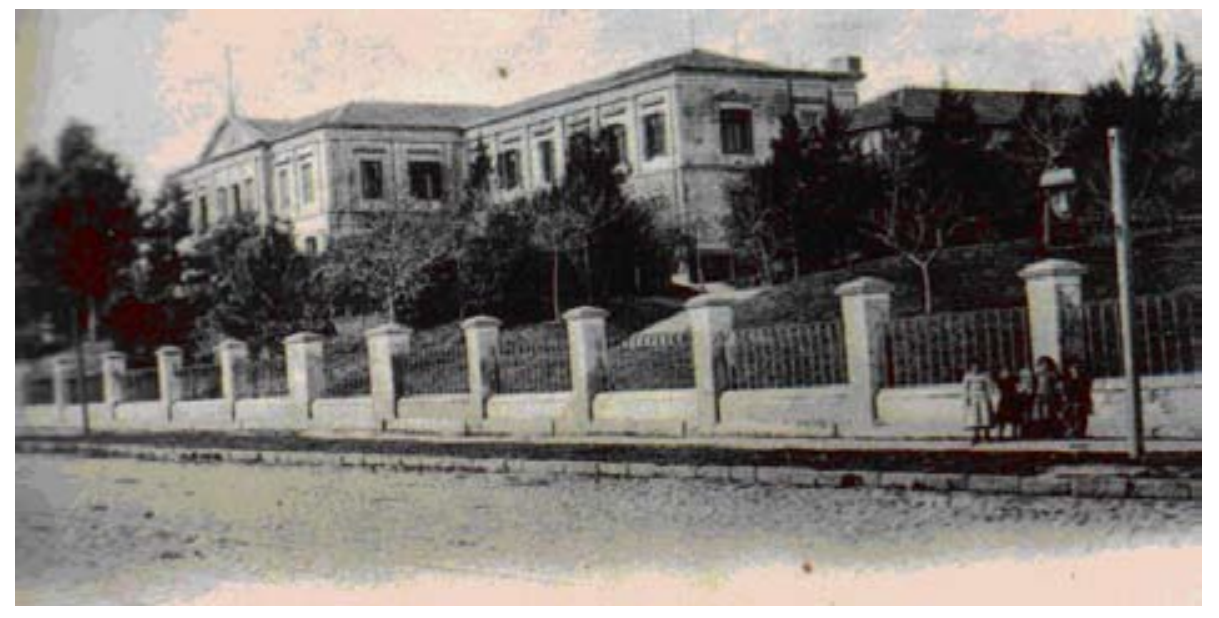

Fig. 4 Vista Hospital Rawson

(Buenos Aires hace cien años, a través de las postales Gobierno de la Ciudad de Buenos Aires) 


\section{IIX) concenso \\ g.

Indexaciones: Repositorio de Revistas UCR, DIALNET, Latindex, REDALYC Directorio y recolector de recursos digitales del Ministerio de Cultura de España, Directory of Open Access Journals.

Diálogos Revista Electrónica de Historia ISSN 1409-469X. Número especial 2008. Dirección web: http://historia.fcs.ucr.ac.cr/dialogos.htm

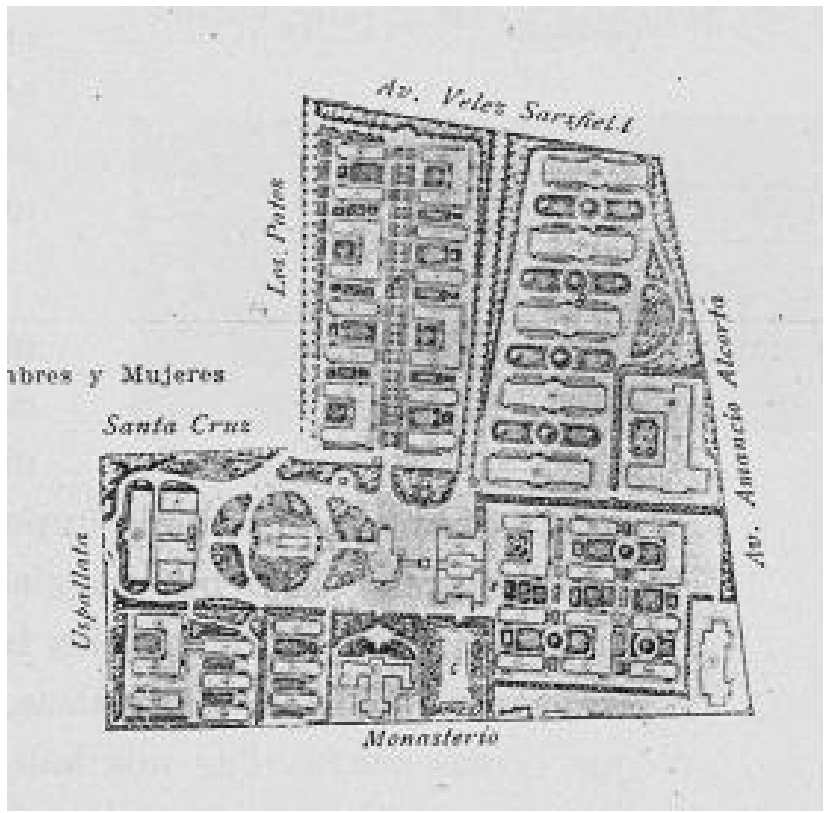

Fig. 5 Planta Hospital Muñiz (Municipalidad de la Capital) 